Bull. Korean Math. Soc. 51 (2014), No. 1, pp. 43-54

http://dx.doi.org/10.4134/BKMS.2014.51.1.043

\title{
EXISTENCE OF SOLUTIONS FOR NONLINEAR EVOLUTION EQUATIONS WITH INFINITE DELAY
}

\author{
QIXIANg Dong And Gang Li
}

\begin{abstract}
This paper is concerned with nonlinear evolution differential equations with infinite delay in Banach spaces. Using Kato's approximating approach, existence and uniqueness of strong solutions are obtained.
\end{abstract}

\section{Introduction}

Let $X$ be a Banach real space with norm $\|\cdot\|$. Consider the nonlinear abstract problem with infinite delay

$$
\left\{\begin{array}{l}
u^{\prime}(t)+A(t) u(t)=F\left(t, u_{t}\right), \quad t \in[0, T] \\
u_{0}=\phi,
\end{array}\right.
$$

where $u:(-\infty, T) \rightarrow X$; for each $t \in[0, T], A(t): D(A(t)) \subset X \rightarrow X ; \phi$ is an element in a phase space (state space) $\mathcal{B}$ of functions mapping $(-\infty, 0]$ into $X$. $F:[0, T] \times \mathcal{B} \rightarrow X$, and $u_{t} \in \mathcal{B}$ defined by $u_{t}(\theta)=u(t+\theta)$ for $\theta \leq 0$. By a strong solution of (1.1), we mean a continuous function $u:(-\infty, T] \rightarrow X$, which is absolutely continuous on $[0, T]$, strongly differentiable for almost all $t \in[0, T]$, and satisfies (1.1). We also say that $u$ is a solution of $(1.1)$ on $[0, T]$.

In the literature devoted to equations with finite delay, the state space is the space of all continuous functions on $[-r, 0], r>0$, endowed with the uniform norm topology. When the delay is unbounded, the selection of the state space $\mathcal{B}$ plays an important role in the study of both qualitative and quantitative theory. A usual choice is a semi-normed space satisfying suitable axioms, which was introduced by Hale and Kato [7]. For a detailed discussion on the topic, we refer to the book by Hino et al. [9]. In the last decades, the theory of functional differential equations of various classes with delay has attracted widespread attention. The development was initiated for equations with finite delay by Travis and Webb $[15,16]$, and Webb $[17,18]$. For later development,

Received March 18, 2012; Revised March 16, 2013.

2010 Mathematics Subject Classification. Primary 35D05, $47 \mathrm{H} 06$.

Key words and phrases. nonlinear evolution equation, m-accretive operator, approximate, strong solution.

This work was supported by the National Natural Science Foundation of China (11271316 and 11201410) and the Natural Science Foundation of Jiangsu Province (BK2012260). 
we mention here the work of some authors [2,3] and [5]. As to the case of infinite delay, an extensive theory is developed for (1.1), where $A(t)$ (or $A(t) \equiv A$ ) is linear. For nonlinear case, we refer the readers to $[6,12,14]$ and [4].

Concerning the case that $A(t)$ are nonlinear, Kartsatos and Parrott [10] showed the existence of strong solution of (1.1) with finite delay. By using Kato's approximating approach, they showed in a straightforward manner that, under certain assumptions on $X, F$ and $A(t), u(t)$, the unique strong solution of (1.1), actually exists as a uniform limit of $\left\{u_{n}(t)\right\}$, where $u_{n}(t), n=1,2, \ldots$ are the unique strongly continuously differentiable solutions of approximating equations

$$
\left\{\begin{array}{l}
u_{n}^{\prime}(t)+A_{n}(t) u_{n}(t)=F\left(t, u_{n_{t}}\right), \quad t \in[0, T] \\
u_{n_{0}}=\phi
\end{array}\right.
$$

here $A_{n}(t)$ are the Yosida approximants.

In this paper, we extend this line of attack to evolution equations with infinite delay. We adopt the phase space introduced by Hale and Kato [7]. By applying the method cited above, we obtain an existence and uniqueness theorem of equation (1.1) with infinite delay. Our result extends and improves those of Kartsatos et al. [10] and Dyson and Bressan [2, 3].

\section{Preliminaries}

In what follows, let $X$ be a real Banach space with $X^{*}$, the dual space of $X$, being uniformly convex. We also assume that $A(t): D(A(t)) \subset X \rightarrow X$, $t \in[0, T]$, are $m$-accretive. We impose the following conditions:

(D1) The domain of $D(A(t)) \equiv D$ is independent of $t$.

(D2) There is a nondecreasing function $L:[0,+\infty) \rightarrow[0,+\infty)$ such that for all $x \in D$ and $s, t \in[0, T]$,

$$
\|A(t) x-A(s) x\| \leq|t-s| L(\|x\|)(1+\|A(s) x\|) .
$$

(D3) There exists a constant $B>0$, such that

$$
\|F(t, \xi)-F(t, \zeta)\| \leq B\|\xi-\zeta\|_{\mathcal{B}}, \xi, \zeta \in \mathcal{B}, t \in[0, T] .
$$

(D4) There exists an increasing function $g:[0,+\infty) \rightarrow[0,+\infty)$ such that

$$
\|F(t, \xi)-F(s, \xi)\| \leq|t-s| g\left(\|\xi\|_{\mathcal{B}}\right), \quad \xi \in \mathcal{B}, t, s \in[0, T] .
$$

We recall the definition of a single-valued operator $A: D(A) \subset X \rightarrow X$ being $m$-accretive. Let $\langle x, y\rangle$ denote the evaluation $y(x)$ for $x \in X, y \in X^{*}$. Define

$$
J(x)=\left\{x^{*} \in X^{*}:\left\langle x, x^{*}\right\rangle=\|x\|^{2}=\left\|x^{*}\right\|^{2}\right\} .
$$

The set $J(x)$ is nonempty for each $x \in X$ by the Hahn Banach theorem. The mapping $J$ is called the duality map of $X$. For a general Banach space $X$, the duality map may be multi-valued. However, if $X^{*}$ is strictly convex, then the duality map $J$ is single-valued. If, moreover, $X^{*}$ is uniformly convex, then $J$ is 
uniformly continuous on bounded subset of $X$. An operator $A: D(A) \subset X \rightarrow$ $X$ is called accretive if for every $x_{1}, x_{2} \in D(A)$, we have

$$
\left\langle A x_{1}-A x_{2}, x_{1}-x_{2}\right\rangle \geq 0 .
$$

An accretive operator $A$ is said to be $m$-accretive if $R(I-\lambda A)=X$ for some $\lambda>0$. If $A$ is $m$-accretive, then $R(I+\lambda A)=X$ for all $\lambda>0$.

Since for each $t \in[0, T], A(t)$ is $m$-accretive, we can define Yosida approximates for $n=1,2, \ldots$ as follows:

$$
\begin{gathered}
J_{n}(t)=(I+(1 / n) A(t))^{-1}, \\
A_{n}(t)=n\left(I-J_{n}\right)(t) .
\end{gathered}
$$

If Condition (C2) is satisfied, then the Yosida approximates are everywhere defined, and

$$
A_{n}(t)=A(t) J_{n}(t)=A(t)(I+(1 / n) A(t))^{-1} .
$$

For the other properties, see, for example, Barbu [1] and Pavel [13].

Definition 2.1. A linear topological space of functions from $(-\infty, 0]$ into $X$, with seminorm $\|\cdot\|_{\mathcal{B}}$, is called an admissible phase space if $\mathcal{B}$ has the following properties:

(A1) There exist a positive constant $H$ and functions $K(\cdot), M(\cdot):[0,+\infty) \rightarrow$ $[0,+\infty)$, with $K$ continuous and $M$ locally bounded, such that for any $a, b \in \mathbb{R}$ and $b>a$, if $x:(-\infty, b] \rightarrow X, x_{a} \in \mathcal{B}$, and $x(\cdot)$ is continuous on $[a, b]$, then for every $t \in[a, b]$, the following conditions hold:

(i) $x_{t} \in \mathcal{B}$;

(ii) $\|x(t)\| \leq H\left\|x_{t}\right\|_{\mathcal{B}}$ for some $H>0$;

(iii) $\left\|x_{t}\right\|_{\mathcal{B}} \leq K(t-a) \sup _{a \leq s \leq t}\|x(s)\|+M(t-a)\left\|x_{a}\right\|_{\mathcal{B}}$.

(A2) For the function $x(\cdot)$ in (A1), $t \mapsto x_{t}$ is a $\mathcal{B}$ valued continuous function for $t \in[a, b]$.

(B) The space $\mathcal{B}$ is complete.

Notice that property (B) is equivalent to say that the space of equivalence classes $\mathcal{B} /\|\cdot\|_{\mathcal{B}}$ is a Banach space.

In the theory of retarded functional differential equations with infinite delay we frequently need some additional properties on the space $\mathcal{B}$ to obtain some results. Next we denote by $C_{00}$ the space of continuous functions from $(-\infty, 0]$ into $X$ with compact support. It is clear from the axioms of phase space that $C_{00} \subset \mathcal{B}$. We consider the following axioms [9]:

(E1) If $\left\{\xi_{n}\right\}$ is a Cauchy sequence in $\mathcal{B}$ which converges to a function $\xi$ uniformly on compact subsets of $(-\infty, 0]$, then $\xi \in \mathcal{B}$ and $\left\|\xi_{n}-\xi\right\|_{\mathcal{B}} \rightarrow 0$ as $n \rightarrow \infty$.

(E2) If a uniformly bounded sequence $\left\{\xi_{n}\right\}$ in $C_{00}$ converges to a function $\xi$ in the compact-open topology then $\xi$ belongs to $\mathcal{B}$ and $\left\|\xi_{n}-\xi\right\|_{\mathcal{B}} \rightarrow 0$ as $n \rightarrow \infty$.

The following property is useful for the existence of strong solution of nonlinear evolution equation. 
Lemma 2.2 ([8]). Assume that $\mathcal{B}$ satisfies axiom (E2). Let $u:(-\infty, a) \rightarrow X$, $a>0$, be a function of class $C^{1}$ such that $u_{0}=\xi$ is bounded and continuous on $(-\infty, 0]$ and $\xi^{\prime}$ is bounded and uniformly continuous. Then the function $[0, a) \rightarrow \mathcal{B}, t \mapsto u_{t}$ is differentiable and $d u_{t} / d t=u_{t}^{\prime}$ for $t<a$.

We also need the following Gronwall's inequality.

Lemma 2.3 (Gronwall's inequality). Let $y(\cdot), \alpha(\cdot), \beta(\cdot)$ be positive functions defined on $[a, b]$, satisfying

$$
y(t) \leq \alpha(t)+\int_{a}^{t} \beta(s) y(s) d s
$$

for all $t \in[a, b]$, then

$$
y(t) \leq \alpha(t) \exp \left(\int_{a}^{t} \beta(s) d s\right)
$$

for all $t \in[a, b]$. In particular, if $\alpha(t)=\alpha=$ constant $>0$, then

$$
y(t) \leq \alpha \exp \left(\int_{a}^{t} \beta(s) d s\right)
$$

for all $t \in[a, b]$. If in addition that $\beta(t)=\beta=$ constant, then

$$
y(t) \leq \alpha \exp (\beta|t-a|)
$$

for all $t \in[a, b]$.

\section{The existence of strong solutions}

Now we state and prove our main result.

Theorem 3.1. Assume that Conditions (D1)-(D4) hold, $\phi \in \mathcal{B}$ is bounded and continuously differentiable such that $\phi(0) \in D$. Then there exists a unique strong solution of (1.1) on $[0, T]$ given by $\lim _{n \rightarrow \infty} u_{n}(t)$, where, for each $n$, $n=1,2, \ldots, u_{n}(\cdot)$ is the unique continuously differentiable solution of $(1.2)$ on $[0, T]$.

The proof of Theorem 3.1 is accomplished by a series of lemmas. We first verify that for each $n, n=1,2, \ldots$, equation (1.2) has a unique continuously differentiable solution $u_{n}(t)$. Then the uniformly boundedness of $\left\{u_{n}(t)\right\}$ and $\left\{u_{n}^{\prime}(t)\right\}$ is established. Finally, we show that the strong limit $u(t)=\lim _{n \rightarrow \infty} u_{n}(t)$ exists uniformly on $[0, T]$, with $u_{0}=\phi$, and satisfies $(1.1)$ for almost all $t \in[0, T]$.

Lemma 3.2. Assume that Conditions (D1)-(D4) hold, $\phi \in \mathcal{B}$ is bounded and continuously differentiable such that $\phi(0) \in D$. Then there exists a unique strongly continuously differentiable solution $u_{n}(t)$ of $(1.2)$ on $[0, T]$. 
Proof. In a manner similar to that of [11] Lemma 4.1, it can be shown that for all $n$ and $x \in X$, we have

$$
\left\|A_{n}(t) x-A_{n}(s) x\right\| \leq|t-s| L_{1}(\|x\|)\left(1+\left\|A_{n}(s) x\right\|\right),
$$

where $L_{1}$ is a nondecreasing function. Inequality (3.1) shows that $A_{n}(t) x$ is Lipschitz continuous in $t$ for every $x \in X$. Also, $A_{n}(t) x$ is uniformly Lipschitz continuous in $x$ for $t \in[0, T]$. Thus, there exists a unique strongly continuously differentiable solution $u_{n}(t)$ of the approximate equation $(1.2)$ on $[0, T]$.

Lemma 3.3. Assume that Conditions (D1)-(D4) hold. Also assume that $\phi(0)=a \in D$ and $\phi$ is continuously differentiable. Then there exists $K>0$, such that $\left\|u_{n}(t)\right\| \leq K$ for all $n=1,2, \ldots$ and $t \in[0, T]$, where $u_{n}(t)$ are the solutions of $(1.2)$.

Proof. First we extend $\phi$ to $t \in(-\infty, T]$ by defining

$$
\phi(t)=\left\{\begin{array}{lc}
\phi(0), & t \in[0, T], \\
\phi(t), & t \in(-\infty, 0] .
\end{array}\right.
$$

Then $\phi_{t} \in \mathcal{B}$ for all $t \in[0, T]$, by $(\mathrm{A} 1)(\mathrm{i})$ (see Definition 2.1). Since $F(\cdot, \phi)$ is continuous on $[0, T]$, we can find $M_{1}>0$, such that $\|F(t, \phi)\| \leq M_{1}$ for all $t \in[0, T]$. By the fact that $u_{n}(t)$ is differentiable on $[0, T]$, the accretiveness of $A_{n}(t)$ and (D3), we have

$$
\begin{aligned}
& \left\langle u_{n}^{\prime}(t), J\left(u_{n}(t)-a\right)\right\rangle \\
= & -\left\langle A_{n}(t) u_{n}(t)-F\left(t, u_{n_{t}}\right), J\left(u_{n}(t)-a\right)\right\rangle \\
= & -\left\langle A_{n}(t) u_{n}(t)-A_{n}(t) a, J\left(u_{n}(t)-a\right)\right\rangle-\left\langle A_{n}(t) a, J\left(u_{n}(t)-a\right)\right\rangle \\
& +\left\langle F\left(t, u_{n_{t}}\right)-F\left(t, \phi_{t}\right), J\left(u_{n}(t)-a\right)\right\rangle-\left\langle F\left(t, \phi_{t}\right), J\left(u_{n}(t)-a\right)\right\rangle \\
\leq & \left(\left\|A_{n}(t) a\right\|+\left\|F\left(t, u_{n_{t}}\right)-F\left(t, \phi_{t}\right)\right\|+\left\|F\left(t, \phi_{t}\right)\right\|\right)\left\|u_{n}(t)-a\right\| \\
\leq & \left(\left\|A_{n}(t) a\right\|+B\left\|u_{n_{t}}-\phi_{t}\right\|_{\mathcal{B}}+M_{1}\right)\left\|u_{n}(t)-a\right\|,
\end{aligned}
$$

From (3.1) we obtain

$$
\begin{aligned}
\left\|A_{n}(t) a-A_{n}(0) a\right\| & \leq t L_{1}(\|a\|)\left(1+\left\|A_{n}(0) a\right\|\right. \\
& \leq T L_{1}(\|a\|)(1+\|A(0) a\|),
\end{aligned}
$$

which yields

$$
\left\|A_{n}(t) a\right\| \leq T L_{1}(\|a\|)(1+\|A(0) a\|)+\|A(0) a\|=K_{1} .
$$

Thus,

$$
\left\langle u_{n}^{\prime}(t), J\left(u_{n}(t)-a\right)\right\rangle \leq\left(K_{1}+B\left\|u_{n_{t}}-\phi\right\|_{\mathcal{B}}+M_{1}\right)\left\|u_{n}(t)-a\right\| .
$$


Since $u_{n}(t)$ is strongly absolutely continuous, so is $\left\|u_{n}(t)-a\right\|$. Thus, $\frac{d}{d t}\left\|u_{n}(t)-a\right\|$ exists a.e. and

$$
\left\|u_{n}(t)-a\right\| \frac{d}{d t}\left\|u_{n}(t)-a\right\|=\left\langle u_{n}^{\prime}(t), J\left(u_{n}(t)-a\right)\right\rangle .
$$

So we obtain from (3.2) that

$$
\begin{aligned}
\frac{d}{d t}\left\|u_{n}(t)-a\right\| & \leq K_{1}+B\left\|u_{n_{t}}-\phi_{t}\right\|_{\mathcal{B}}+M_{1} \\
& =K_{2}+B\left\|u_{n_{t}}-\phi_{t}\right\|_{\mathcal{B}},
\end{aligned}
$$

where $K_{2}=K_{1}+M_{1}$. Now we integrate (3.3) to obtain

$$
\left\|u_{n}(t)-a\right\| \leq K_{2} t+B \int_{0}^{t}\left\|u_{n_{s}}-\phi_{s}\right\|_{\mathcal{B}} d s, \quad t \in[0, T] .
$$

Set $K_{T}=\sup _{0 \leq t \leq T} K(t)$, where $K(\cdot)$ is the function in (A1). Then by (A1)(iii), we have

$$
\begin{aligned}
\left\|u_{n_{t}}-\phi_{t}\right\|_{\mathcal{B}} & \leq K(t) \sup _{0 \leq \tau \leq t}\left\|u_{n}(\tau)-\phi(\tau)\right\| \\
& \leq K_{T} \sup _{0 \leq \tau \leq t}\left\|u_{n}(\tau)-a\right\| .
\end{aligned}
$$

Hence, from (3.4) we get

$$
\begin{aligned}
\left\|u_{n_{t}}-\phi_{t}\right\|_{\mathcal{B}} & \leq K_{T} K_{2} t+B K_{T} \sup _{0 \leq \tau \leq t} \int_{0}^{\tau}\left\|u_{n_{s}}-\phi_{s}\right\|_{\mathcal{B}} d s \\
& \leq K_{T} K_{2} t+B K_{T} \int_{0}^{t}\left\|u_{n_{s}}-\phi_{s}\right\|_{\mathcal{B}} d s, \quad t \in[0, T] .
\end{aligned}
$$

An application of Gronwall's inequality in (3.5) yields

$$
\left\|u_{n_{t}}-\phi_{t}\right\|_{\mathcal{B}} \leq K_{T} K_{2} t \exp \left(K_{T} B t\right), \quad t \in[0, T]
$$

and hence

$$
\left\|u_{n_{t}}-\phi_{t}\right\|_{\mathcal{B}} \leq K_{T} K_{2} T \exp \left(K_{T} B T\right), \quad t \in[0, T]
$$

Therefor, by (A1)(ii),

$$
\left\|u_{n}(t)-a\right\| \leq H\left\|u_{n_{t}}-\phi_{t}\right\|_{\mathcal{B}}, \quad t \in[0, T]
$$

from which follows the uniformly boundedness of $\left\{u_{n}(t)\right\}$, taking

$$
K=K_{T} K_{2} T \exp \left(K_{T} B T\right)+\|a\| \text {. }
$$

Remark 3.4. From the proof of Lemma 3.3, it is easily seen that the constant $K$ defined above is also such that $\left\|u_{n_{t}}-\phi_{t}\right\|_{\mathcal{B}} \leq K,\left\|u_{n_{t}}\right\|_{\mathcal{B}} \leq K$, and $\left\|u_{n}(t)\right\| \leq K$ for all $n=1,2, \ldots$ and $t \in[0, T]$.

Similar to Lemma 2.4 in [10], we have the following 
Lemma 3.5. Let $w \in C_{1}([0, T] ; X)$ be given. Then for any $s \in[0, T]$,

$$
\lim _{h \rightarrow 0+} \sup _{0 \leq \theta \leq s}\|w(\theta+h)-w(\theta)\| / h
$$

exists and equals

$$
\sup _{0 \leq \theta \leq s}\left\|w^{\prime}(\theta)\right\|
$$

Lemma 3.6. Assume that the conditions of Lemma 3.3 hold. Then there exists $N>0$, such that $\left\|u_{n}^{\prime}(t)\right\| \leq N$ for all $n=1,2, \ldots$ and $t \in[0, T]$. Here $u_{n}(t)$ are the solutions of (1.2).

Proof. Let $z_{n}(t)=u_{n}(t+h)-u_{n}(t),(0<h<t)$. Then,

$$
\begin{aligned}
\left\|z_{n}(t)\right\| \frac{d}{d t}\left\|z_{n}(t)\right\|= & \left\langle z_{n}^{\prime}(t), J\left(z_{n}(t)\right\rangle\right. \\
= & -\left\langle A_{n}(t+h) u_{n}(t+h)-A_{n}(t) u_{n}(t), J\left(z_{n}(t)\right\rangle\right. \\
& +\left\langle F\left(t+h, u_{n_{t+h}}\right)-F\left(t, u_{n_{t}}\right), J\left(z_{n}(t)\right\rangle\right. \\
= & -\left\langle A_{n}(t+h) u_{n}(t+h)-A_{n}(t+h) u_{n}(t), J\left(z_{n}(t)\right\rangle\right. \\
& +\left\langle A_{n}(t) u_{n}(t)-A_{n}(t+h) u_{n}(t), J\left(z_{n}(t)\right\rangle\right. \\
& +\left\langle F\left(t+h, u_{n_{t+h}}\right)-F\left(t+h, u_{n_{t}}\right), J\left(z_{n}(t)\right\rangle\right. \\
& +\left\langle F\left(t+h, u_{n_{t}}\right)-F\left(t, u_{n_{t}}\right), J\left(z_{n}(t)\right\rangle\right. \\
\leq & \left(h L_{1}\left(\left\|u_{n}(t)\right\|\right)\left(1+\left\|A_{n}(t) u_{n}(t)\right\|\right)\right. \\
& \left.+B\left\|u_{n_{t+h}}-u_{n_{t}}\right\| \mathcal{B}+h g\left(\left\|u_{n_{t}}\right\|_{\mathcal{B}}\right)\right)\left\|z_{n}(t)\right\|
\end{aligned}
$$

a.e. on $[0, T]$. Here we have used the accretiveness of $A_{n}(t+h)$, inequality (3.1), conditions (D3) and (D4).

By Remark 3.4, there exists $K^{\prime}>0$, such that $\left\|u_{n_{t}}-\phi_{t}\right\|_{\mathcal{B}} \leq K^{\prime},\left\|u_{n_{t}}\right\|_{\mathcal{B}} \leq$ $K^{\prime}$, and $\left\|u_{n}(t)\right\| \leq K^{\prime}$ for all $n=1,2, \ldots$ and $t \in[0, T]$. Since

$$
\begin{aligned}
\left\|A_{n}(t) u_{n}(t)\right\| & \leq\left\|u_{n}^{\prime}(t)\right\|+\left\|F\left(t, u_{n_{t}}\right)\right\| \\
& \leq\left\|u_{n}^{\prime}(t)\right\|+\| F\left(t, u_{n_{t}}-F\left(t, \phi_{t}\right)\|+\| F\left(t, \phi_{t}\right) \|\right. \\
& \leq\left\|u_{n}^{\prime}(t)\right\|+B\left\|u_{n_{t}}-\phi_{t}\right\|_{\mathcal{B}}+M_{1} \\
& \leq\left\|u_{n}^{\prime}(t)\right\|+B K^{\prime}+M_{1},
\end{aligned}
$$

inequality (3.7) yields

$$
\frac{d}{d t}\left\|z_{n}(t)\right\| \leq h C_{1}+h C_{2}\left\|u_{n}^{\prime}(t)\right\|+B\left\|u_{n_{t+h}}-u_{n_{t}}\right\|_{\mathcal{B}}
$$

where $C_{1}=L_{1}\left(K^{\prime}\right)\left(1+B K^{\prime}+M_{1}\right)+g\left(K^{\prime}\right), C_{2}=L_{1}\left(K^{\prime}\right)$. An integration above gives

$$
\begin{aligned}
\left\|z_{n}(t)\right\| \leq & \left\|z_{n}(0)\right\|+h C_{1} T+h C_{2} \int_{0}^{t}\left\|u_{n}^{\prime}(s)\right\| d s \\
& +B \int_{0}^{t}\left\|u_{n_{s+h}}-u_{n_{s}}\right\|_{\mathcal{B}} d s
\end{aligned}
$$


that is

$$
\begin{aligned}
& \left\|u_{n}(t+h)-u_{n}(t)\right\| / h \leq\left\|u_{n}(h)-u_{n}(0)\right\| / h+C_{1} T+C_{2} \int_{0}^{t}\left\|u_{n}^{\prime}(s)\right\| d s \\
& +B \int_{0}^{t}\left\|u_{n_{s+h}}-u_{n_{s}}\right\|_{\mathcal{B}} / h d s .
\end{aligned}
$$

Set $K_{T}=\sup _{0 \leq s \leq T} K(s)$ and $M_{T}=\sup _{0 \leq s \leq T} M(s)$, where $K(\cdot)$ and $M(\cdot)$ are the functions in (A1). Make use of (A1) to obtain

$$
\begin{gathered}
\int_{0}^{t}\left\|u_{n_{s+h}}-u_{n_{s}}\right\|_{\mathcal{B}} / h d s \leq K_{T} \int_{0}^{t} \sup _{0 \leq \theta \leq s}\left\|u_{n}(\theta+h)-u_{n}(\theta)\right\| / h d s \\
\quad+M_{T} \int_{0}^{t}\left\|u_{n_{h}}-\phi_{0}\right\|_{\mathcal{B}} / h d s \\
\leq K_{T} \int_{0}^{t} \sup _{0 \leq \theta \leq s}\left\|u_{n}(\theta+h)-u_{n}(\theta)\right\| / h d s \\
+M_{T} T\left\|u_{n_{h}}-\phi_{0}\right\|_{\mathcal{B}} / h d s .
\end{gathered}
$$

From inequality (3.5) we get that

$$
\begin{aligned}
\left\|u_{n_{h}}-\phi_{0}\right\|_{\mathcal{B}} / h & \leq\left\|u_{n_{h}}-\phi_{h}\right\|_{\mathcal{B}} / h+\left\|\phi_{h}-\phi_{0}\right\|_{\mathcal{B}} / h \\
& \leq K_{T} K_{2} \exp \left(B K_{T} T\right)+\left\|\phi_{h}-\phi_{0}\right\|_{\mathcal{B}} / h .
\end{aligned}
$$

By Lemma 3.5, we have

$$
\lim _{h \rightarrow 0+} \int_{0}^{t} \sup _{0 \leq \theta \leq s}\left\|u_{n}(\theta+h)-u_{n}(\theta)\right\| / h d s=\int_{0}^{t} \sup _{0 \leq \theta \leq s}\left\|u_{n}^{\prime}(\theta)\right\| d s .
$$

Also we have that

$$
\begin{aligned}
\lim _{h \rightarrow 0+}\left\|u_{n}(h)-u_{n}(0)\right\| / h & =\left\|u_{n}^{\prime}(0)\right\| \\
& \leq\left\|A_{n}(0) u_{n}(0)\right\|+\|F(0, \phi)\| \\
& \leq\|A(0) a\|+M_{1} .
\end{aligned}
$$

An application of Lemma 2.2 yields that $\left\|\phi_{h}-\phi\right\| / h$ is bounded, i.e., there exists $M_{2}>0$ such that $\left\|\phi_{h}-\phi\right\| / h \leq M_{2}$ for sufficiently small $h$. Thus we can get from (3.8)-(3.12) that

$$
\begin{aligned}
\left\|u_{n}^{\prime}(t)\right\| & =\lim _{h \rightarrow 0+}\left\|u_{n}(t+h)-u_{n}(t)\right\| / h \\
& \leq C_{3}+C_{4} \int_{0}^{t} \sup _{0 \leq \theta \leq s}\left\|u_{n}^{\prime}(\theta)\right\| d s
\end{aligned}
$$

for all $t \in[0, T]$, where $C_{3}=\|A(0) a\|+M_{1}+C_{1} T+B M_{T} T K_{T} K_{2}\left(\exp \left(B K_{T} T\right)+\right.$ $\left.M_{2}\right), C_{4}=C_{2}+B K_{T}$, and hence

$$
\sup _{0 \leq \theta \leq t}\left\|u_{n}^{\prime}(\theta)\right\| \leq C_{3}+C_{4} \int_{0}^{t} \sup _{0 \leq \theta \leq s}\left\|u_{n}^{\prime}(\theta)\right\| d s
$$


for all $t \in[0, T]$. Therefore, $\left\{u_{n}^{\prime}(t)\right\}$ is uniformly bounded, by Gronwall's inequality.

Lemma 3.7. Assume that the conditions of Lemma 3.3 hold. Then the strong limit $\lim _{n \rightarrow \infty} u_{n}(t)$ exists uniformly on $[0, T]$.

Proof. Let $x_{m n}(t)=u_{m}(t)-u_{n}(t)$. Then we have, a.e. $t$

$$
\begin{aligned}
\frac{1}{2} \frac{d}{d t}\left\|x_{m n}(t)\right\|^{2}= & \left\langle x_{m n}^{\prime}(t), J\left(x_{m n}(t)\right\rangle\right. \\
= & -\left\langle A_{m}(t) u_{m}(t)-A_{n}(t) u_{n}(t), J\left(x_{m n}(t)\right)\right\rangle \\
& +\left\langle F\left(t, u_{m_{t}}\right)-F\left(t, u_{n_{t}}\right), J\left(x_{m n}(t)\right)\right\rangle .
\end{aligned}
$$

Since $A_{m}(t) u_{m}(t)=A(t) J_{m}(t) u_{m}(t), A_{n}(t) u_{n}(t)=A(t) J_{n}(t) u_{n}(t)$ and $A(t)$ is accretive,

$$
\left\langle A_{m}(t) u_{m}(t)-A_{n}(t) u_{n}(t), J\left(y_{m n}(t)\right)\right\rangle \geq 0,
$$

where $y_{m n}(t)=J_{m}(t) u_{m}(t)-J_{n}(t) u_{n}(t)$. Adding (3.13) and (3.14), we get a.e. $t$

$$
\begin{aligned}
\frac{1}{2} \frac{d}{d t}\left\|x_{m n}(t)\right\|^{2} \leq & \left\langle A_{m}(t) u_{m}(t)-A_{n}(t) u_{n}(t), J\left(y_{m n}(t)\right)-J\left(x_{m n}(t)\right)\right\rangle \\
& +\left\langle F\left(t, u_{m_{t}}\right)-F\left(t, u_{n_{t}}\right), J\left(x_{m n}(t)\right)\right\rangle \\
\leq & \left\|A_{m}(t) u_{m}(t)-A_{n}(t) u_{n}(t)\right\|\left\|J\left(y_{m n}(t)\right)-J\left(x_{m n}(t)\right)\right\| \\
& +B\left\|u_{m_{t}}-u_{n_{t}}\right\|_{\mathcal{B}} \cdot\left\|u_{m}(t)-u_{n}(t)\right\| .
\end{aligned}
$$

By the uniform boundedness of $\left\{u_{n}(t)\right\}$ and $\left\{u_{n}^{\prime}(t)\right\}$, we get

$$
\begin{aligned}
\left\|A_{n}(t) u_{n}(t)\right\| & \leq\left\|u_{n}^{\prime}(t)\right\|+\left\|F\left(t, u_{n_{t}}\right)\right\| \\
& \leq\left\|u_{n}^{\prime}(t)\right\|+B\left\|u_{n_{t}}-\phi\right\|_{\mathcal{B}}+\|F(t, \phi)\| \\
& \leq N+B K^{\prime}+M_{1}=M_{0} .
\end{aligned}
$$

Hence, by (3.15), (3.16) and (A1)(ii),

$$
\begin{aligned}
\frac{1}{2} \frac{d}{d t}\left\|x_{m n}(t)\right\|^{2} \leq & 2 M_{0}\left\|J\left(y_{m n}(t)\right)-J\left(x_{m n}(t)\right)\right\| \\
& +B\left\|u_{m_{t}}-u_{n_{t}}\right\|_{\mathcal{B}}\left\|u_{m}(t)-u_{n}(t)\right\| \\
\leq & 2 M_{0}\left\|J\left(y_{m n}(t)\right)-J\left(x_{m n}(t)\right)\right\|+H B\left\|u_{m_{t}}-u_{n_{t}}\right\|_{\mathcal{B}}^{2} .
\end{aligned}
$$

From the absolute continuity of $\left\|x_{m n}(t)\right\|^{2}$ and the fact that $x_{m n}(0)=0$, we obtain

$$
\begin{aligned}
\left\|x_{m n}(t)\right\|^{2} & =\left\|u_{m}(t)-u_{n}(t)\right\|^{2} \\
& \leq 4 M_{0} \int_{0}^{T}\left\|J\left(y_{m n}(s)\right)-J\left(x_{m n}(s)\right)\right\| d s+4 B H \int_{0}^{t}\left\|u_{m_{s}}-u_{n_{s}}\right\|_{\mathcal{B}}^{2} d s .
\end{aligned}
$$

By (A1)(ii), we get

$$
\left\|u_{m_{t}}-u_{n_{t}}\right\|_{\mathcal{B}}^{2} \leq K_{T}^{2}\left\|u_{m}(t)-u_{n}(t)\right\|^{2}
$$




$$
\begin{aligned}
\leq & 4 M_{0} K_{T}^{2} \int_{0}^{T}\left\|J\left(y_{m n}(s)\right)-J\left(x_{m n}(s)\right)\right\| d s \\
& +4 B H K_{T}^{2} \int_{0}^{t}\left\|u_{m_{s}}-u_{n_{s}}\right\|_{\mathcal{B}}^{2} d s .
\end{aligned}
$$

An application of Gronwall's inequality yields

$$
\left\|u_{m_{t}}-u_{n_{t}}\right\|_{\mathcal{B}}^{2} \leq C_{4} \int_{0}^{T}\left\|J\left(y_{m n}(s)\right)-J\left(x_{m n}(s)\right)\right\| d s
$$

for all $t \in[0, T]$, where $C_{4}=4 M_{0} K_{T}^{2} \exp \left(4 B H K_{T}^{2} T\right)$.

Now we observe that $\left\|x_{m n}(t)\right\|=\left\|u_{m}(t)-u_{n}(t)\right\| \leq 2 K^{\prime}$. Also, by (3.16) and the definition of $J_{n}(t)$, we have

$$
\begin{aligned}
\left\|y_{m n}(t)-x_{m n}(t)\right\| & \leq\left\|J_{m}(t) u_{m}(t)-u_{m}(t)\right\|+\left\|J_{n}(t) u_{n}(t)-u_{n}(t)\right\| \\
& \leq \frac{1}{m}\left\|A_{m}(t) u_{m}(t)\right\|+\frac{1}{n}\left\|A_{n}(t) u_{n}(t)\right\| \\
& \leq \frac{m+n}{m n} M_{0},
\end{aligned}
$$

which tends to zero as $m, n \rightarrow \infty$. By the uniform continuity of $J$ on bounded subset of $X$, given $\varepsilon>0$ we have that, $\left\|J\left(y_{m n}(t)\right)-J\left(x_{m n}(t)\right)\right\|<\varepsilon, 0 \leq t \leq T$, for all sufficiently large $m, n$. Thus, from (3.16), we have

$$
\lim _{n \rightarrow \infty} u_{n_{t}}=u_{t}
$$

uniformly in $t \in[0, T]$. Since

$$
\left\|u_{m}(t)-u_{n}(t)\right\| \leq K_{T}\left\|u_{m_{t}}-u_{n_{t}}\right\|_{\mathcal{B}},
$$

the above limit implies that $\left\|u_{m}(t)-u_{n}(t)\right\| \rightarrow 0$ uniformly in $t \in[0, T]$ as $m, n \rightarrow \infty$. This implies in turn that

$$
\lim _{n \rightarrow \infty} u_{n}(t)=u(t)
$$

exists uniformly in $t \in[0, T]$.

Since $u_{n}(t)$ is Lipschitz continuous with Lipschitz constant independent of $n$ $\left(\left\|u_{n}^{\prime}(t)\right\| \leq N\right)$, the limit $u(t)$ is also Lipschitz continuous with $u(0)=\phi(0)=a$. From $u_{n_{t}} \rightarrow u_{t}$ we also conclude that $u_{0}=\phi$.

Lemma 3.8. Let the conditions of Lemma 3.3 hold. If $u(t)=\lim _{n \rightarrow \infty} u_{n}(t)$ (Lemma 3.7), then $u(t) \in D$ for all $t \in[0, T]$, and $A(t) u(t)$ is bounded and weakly continuous. Moreover, the function $-A(t) u(t)+F\left(t, u_{t}\right)$ is Bochner integrable and $u(t)$ is an indefinite integral of $-A(t) u(t)+F\left(t, u_{t}\right)$. The strong derivative $u^{\prime}(t)$ also exists a.e. $t$, and equals $-A(t) u(t)+F\left(t, u_{t}\right)$.

The proof of Lemma 3.8 follows as in Kato's paper [11], and so it is omitted. Now the proof of Theorem 3.1 has been accomplished. 
Let $\phi \in \mathcal{B}$ satisfy the conditions of Theorem 3.1. Then, there is a corresponding unique solution of (1.1), that we denote by $u(t, \phi)$.

Theorem 3.9. Assume that the conditions of Theorem 3.1 hold. Then the mapping $\phi \mapsto u(t, \phi)$ is a Lipschitz continuous function of $\phi$ in the sense that, there exists a constant $L>0$, such that

$$
\|u(t, \phi)-u(t, \psi)\| \leq L\|\phi-\psi\|_{\mathcal{B}}
$$

for all $t \in[0, T]$ and $\phi, \psi \in \mathcal{B}$ satisfying the conditions of Theorem 3.1.

Proof. We denote $u(t)=u(t, \phi), v(t)=u(t, \psi)$, and $x(t)=u(t)-v(t)$. Then $x(t)$ is Lipschitz continuous, by Lemma 3.6, and hence $\|x(t)\|$ is differentiable a.e. on $[0, T]$. Thus we have

$$
\begin{aligned}
\|x(t)\| \frac{d}{d t}\|x(t)\|= & -\langle A(t) u(t)-A(t) v(t), J(x(t))\rangle \\
& +\left\langle F\left(t, u_{t}\right)-F\left(t, v_{t}\right), J(x(t))\right\rangle \\
\leq & B\left\|u_{t}-v_{t}\right\|_{\mathcal{B}}\|u(t)-v(t)\|,
\end{aligned}
$$

a.e., and so

$$
\frac{d}{d t}\|x(t)\| \leq B\left\|u_{t}-v_{t}\right\|_{\mathcal{B}}
$$

a.e. on $[0, T]$. An integration of this inequality gives

$$
\begin{aligned}
\|x(t)\|=\|u(t)-v(t)\| & \leq\|\phi(0)-\psi(0)\|+B \int_{0}^{t}\left\|u_{s}-v_{s}\right\|_{\mathcal{B}} d s \\
& \leq H\|\phi-\psi\|_{\mathcal{B}}+B \int_{0}^{t}\left\|u_{s}-v_{s}\right\|_{\mathcal{B}} d s
\end{aligned}
$$

for all $t \in[0, T]$. From (A1)(iii) and (3.18), we have

$$
\begin{aligned}
\left\|u_{t}-v_{t}\right\|_{\mathcal{B}} & \leq K_{T} \sup _{0 \leq \theta \leq t}\|u(\theta)-v(\theta)\|+M_{T}\|\phi-\psi\|_{\mathcal{B}} \\
& \leq\left(K_{T} H+M_{T}\right)\|\phi-\psi\|_{\mathcal{B}}+K_{T} B \int_{0}^{t}\left\|u_{s}-v_{s}\right\|_{\mathcal{B}} d s .
\end{aligned}
$$

Consequently,

$$
\left\|u_{t}-v_{t}\right\|_{\mathcal{B}} \leq C_{5}\|\phi-\psi\|_{\mathcal{B}}
$$

for all $t \in[0, T]$, by Gronwall's inequality, where $C_{5}=\left(K_{T} H+M_{T}\right) \exp \left(K_{T} B T\right)$. Therefor, by (A1)(ii), we have

$$
\|u(t)-v(t)\| \leq H C_{5}\|\phi-\psi\|_{\mathcal{B}}
$$

for all $t \in[0, T]$. 


\section{References}

[1] V. Barbu, Nonlinear Semigroups and Differential Equations in Banach Spaces, Noordhoff, Leyden, 1976.

[2] J. Dyson and R. V. Bressan, Functional differential equations and non-linear evolution operators, Proc. Roy. Soc. Edinburgh Ser. A 75 (1975/1976), no. 3, 223-234.

[3] - Semigroups of translations associated with functional and functionaldifferential equations, Proc. Roy. Soc. Edinburgh Sect. A 82 (1978/79), no. 3-4, 171-188.

[4] Z. Fan, Existence and continuous dependence results for nonlinear differential inclusions with infinite delay, Nonlinear Anal. 69 (2008), no. 8, 2379-2392.

[5] W. Fitzgibbon, Representation and approximation of solutions to semilinear Volterra equations with delay, J. Differential Equations 32 (1979), no. 2, 233-249.

[6] C. Gori, V. Obukhovskii, M. Ragni, and P. Rubbioni, Existence and continuous dependence results for semilinear functional differential inclusions with infinite delay, Nonlinear Anal. 51 (2002), 765-782.

[7] J. K. Hale and J. Kato, Phace space for retarded equations with infinite delay, Funkcial. Ekvac. 21 (1978), no. 1, 11-41.

[8] H. R. Henríquez, Differentiability of solutions of second-order functional differential equations with unbounded delay, J. Math. Anal. Appl. 280 (2003), no. 2, 284-312.

[9] Y. Hino, S. Murakami, and T. Naito, Functional Differential Equations with Infinite Delay, in Lecture Notes in Math. Vol. 1473, Springer-Verlag, Berlin, 1991.

[10] A. G. Kartsatos and M. E. Parrott Convergence of the Kato approximants for evolution equations involving functional perturbations, J. Differential Equations 47 (1983), no. 3, $358-377$.

[11] T. Kato, Nonlinear semigroups and evolution equations, J. Math. Soc. Japan 19 (1967), 508-520.

[12] J. Liang and T. J. Xiao, The Cauchy problem for nonlinear abstract functional differential equations with infinite delay, Comput. Math. Appl. 40 (2000), no. 6-7, 693-703.

[13] N. H. Pavel, Nonlinear evolution operators and semigroups, in Lecture Notes in Mathematics, Vol. 1260, Springer-Verlag, Berlin, 1987.

[14] K. Schumacher, Existence and continuous dependence for functional differential equations with unbounded delay, Arch. Ration. Mech. Anal. 67 (1978), no. 4, 315-335.

[15] C. C. Travis and G. F. Webb, Existence and stability for partial functional differential equations, Trans. Amer. Math. Soc. 200 (1974), 395-418.

[16] _ Existence, stability and compactness in the $\alpha$-norm for partial functional differential equations, Trans. Amer. Math. Soc. 240 (1978), 129-143.

[17] G. F. Webb, Autonomous nonlinear functional differential equations and nonlinear semigroups, J. Math. Anal. Appl. 46 (1974), 1-12.

[18] _ Asymptotic stability for abstract nonlinear functional differential equations, Proc. Amer. Math. Soc. 54 (1976), 225-230.

QIXIANG DONG

School of Mathematical Science

YANGZHOU UNIVERSITY

YANGzhou 225002, P. R. China

E-mail address: qxdongyz@yahoo.com.cn

GANG LI

School of Mathematical Science

YANGZHOU UNIVERSITY

YANGZhou 225002, P. R. ChinA

E-mail address: gli@yzu.edu.cn 